\title{
VIVÊNCIA FAMILIAR DA CRIANÇA HOSPITALIZADA COM CÂNCER
}

\section{FAMILY HISTORY OF HOSPITALIZED CHILDREN WITH CANCER}

\section{VIVENCIA FAMILIAR DEL NIÑO HOSPITALIZADO CON CÂNCER}

\author{
Rosana Fidelis Coelho Vieira ${ }^{1}$, Fátima Helena do Espírito Santo², Fernanda Ferreira da Silva Lima
}

\section{RESUMO}

Objetivo: identificar os indicadores científicos sobre a vivência familiar da criança hospitalizada com câncer. Métodos: revisão integrativa de literatura, nas bases de dados Literatura Latino-Americana e do Caribe em Ciências da Saúde, Medical Literature Analysis and Retrieval System On-line, Cumulative Index to Nursingand Allied Health e Base de Dados em Enfermagem. Resultados: dezenove artigos, selecionados dentre os 413 publicados, entre 2010 e 2019, cujos principais sintomas analisados foram categorizados em: sentimentos do familiar da criança com câncer; necessidades do familiar da criança com câncer; enfermagem e família no enfrentamento da doença. Conclusão: constata-se o papel relevante da enfermagem, ao contribuir com ações que atendam às dimensões clínicas, afetivas, sociais e emocionais das famílias que precisam ser amparadas e acolhidas, para resgatarem sua autonomia, construindo com elas relações de confiança, fortalecendo-as para o enfrentamento da doença.

Descritores: Criança Hospitalizada; Família; Neoplasias; Enfermagem.

\section{ABSTRACT}

Objective: to identify the scientific evidences about the family experience of hospitalized children with cancer. Methods: integrative literature review, Latin American and Caribbean Literature in Health Sciences, Medical Literature Analysis and Retrieval System Online, Cumulative Index to Nursing and Allied Health and Nursing Database. Results: a total of 19 articles were selected from 413 publications from 2010 to 2019, in which the main analyzed evidences were categorized into: feelings of the family member of the child with cancer; needs of the family member of the child with cancer; nursing and family in coping with the disease. Conclusion: We found scientific evidence on the family theme of children hospitalized with cancer, highlighting the relevant role of nursing. Furthermore, it contributes to actions related to the clinical, affective, social and emotional dimensions of families who need to be supported and accepted to rescue their autonomy, building trusting relationships and strengthening them to deal with the disease.

Descriptors: Child, Hospitalized; Family; Neoplasms; Nursing.

\section{RESUMEN}

Objetivo: identificar las evidencias científicas sobre la vivencia familiar de niños hospitalizados con cáncer. Métodos: revisión integradora de literatura, en las bases de datos Literatura Latinoamericana y Caribeña en Ciencias de la Salud, Medical Literature Analysis and Retrieval System On-line, Cumulative Index to Nursing and Allied Health y Base de Datos en Enfermería. Resultados: diecinueve artículos fueron seleccionados de 413 publicados entre 2010 y 2019, cuyas principales evidencias analizadas se clasificaron en: sentimientos del familiar del niño con cáncer; necesidades del familiar del niño con cáncer; enfermería y familia en el enfrentamiento de la enfermedad. Conclusión: se encontró evidencia científica sobre el tema familiar de los niños hospitalizados con cáncer, destacando el rol relevante de la enfermería, contribuyendo a laacciones que atienden las dimensiones clínicas, afectivas, sociales y emocionales de las familias que necesitan apoyo y recogida para rescatar su autonomía, construyendo relaciones de confianza, fortaleciéndolas para enfrentar la enfermedad.

Descriptores: Niño Hospitalizado; Familia; Neoplasias; Enfermería.

${ }^{1}$ Mestre em Enfermagem. Enfermeira do Instituto Nacional do Câncer (INCA). 2Doutora em Enfermagem. Professora Associada do Departamento de Enfermagem Médico Cirúrgica da Escola de Enfermagem Aurora de Afonso Costa da Universidade Federal Fluminense (EEAAC/ UFF). ${ }^{3}$ Mestre em Patologia Enfermeira do Instituto Nacional do Câncer (INCA).

\section{Como citar este artigo:}

Vieira RFC, Santo FHE. Lima FFS. Vivência familiar da criança hospitalizada com câncer. Revista de Enfermagem do Centro Oeste Mineiro. 2020;10:e3546. [Access ]; Available in: DOI: http//doi.org/10.19175/recom.v10i0.3546 


\section{INTRODUÇÃO}

No Brasil, o câncer representa a primeira causa de morte por doença entre crianças e adolescentes na faixa etária de um a 19 anos e, conforme dados do Sistema de Informação de Mortalidade, na faixa 0 a 4 anos, ocupa a oitava posição entre as causas de óbito e de 5 a 19 é a principal causa ${ }^{(1)}$.

Considerando o Índice de Desenvolvimento Humano (IDH), é possível projetar que, em países com baixo índice desse indicador, ocorra um aumento do câncer em $112 \%$ e, em países com médio IDH, a taxa aumente em $86 \%$ entre 2012 e 2035, revelando uma proporcionalidade de casos de câncer maior nos países de IDH mais baixo ${ }^{(2)}$.

É, de fato, um problema mundial que vem trazendo preocupações no que diz respeito à prevenção, diagnóstico precoce, tratamento e reabilitação. A expectativa de câncer, no Brasil, publicada em 2019, para o triênio 2020-2022,é de 450 mil casos novos, para cada ano, com exceção do câncer de pele não melanoma. Depreende-se, para o mesmo período, a ocorrência de 8460 casos novos em crianças e adolescentes na faixa etária até os $19 \operatorname{anos}^{(3)}$.

Ainda, de acordo com a estimativa, para o ano de 2020, a taxa bruta de incidência de casos novos de câncer, em crianças e adolescentes, será de 3690, na região Sudeste e 2190 na região Nordeste. Essas regiões apresentarão os maiores números de casos novos ${ }^{(3)}$.

O adoecimento da criança e sua hospitalização acarretam diferentes sentimentos no familiar cuidador, principalmente, pelas rotinas desconhecidas e aparelhagens, despertando-lhes medo, expectativas e incertezas e podem até mesmo lhes proporcionar traumas $^{(4-5)}$. As mães, há poucas décadas, não podiam acompanhar seus filhos, sendo afastadas, no momento difícil para a criança, a qual se encontrava doente, fora do seu lar, em um ambiente novo e estranho, sem a presença da família(6).

Porém é, na família, que a criança, durante a hospitalização,ampara-se à procura de afeto, proteção e apoio. Assim sendo, o cuidado à criança,quando compartilhado com a equipe de enfermagem e o familiar cuidador, pode ser favorável para que as famílias vivenciem momentos menos desgastantes na hospitalização. Torna-se essencial o incentivo com ações integradas e a inclusão da família no cuidado à criança, para um melhor controle da situação e diminuir seu sofrimento. A família é uma peça fundamental no tratamento pela sua capacidade de reorganização e ação ${ }^{(7-9)}$.

Em geral, é a mãe quem mais acompanha o filho, em um processo de adoecimento e hospitalização, gerando,na criança e adolescente, diferentes sentimentos, como o de culpa, dor, choque, impotência pela impossibilidade de fazer algo, além de se sentir responsável pelo filho. Paralelo à essa realidade, há um desequilíbrio de suas atividades habituais mesmo que de forma transitória. ${ }^{(10-11)} \cdot \mathrm{Na}$ internação de pacientes pediátricos, a permanência do acompanhante é necessária, e os estabelecimentos de saúde devem se adequar à presença dos pais ou responsáveis, durante o tempo de hospitalização, garantindo-lhes acesso igualitário, conforme prevê o artigo 12 do Estatuto da Criança e do Adolescente ${ }^{(12)}$.

A presença do familiar acompanhante tem se tornado cada vez mais frequente independente da faixa etária e do nível de dependência do paciente ${ }^{(13)}$. Vem despertando diferentes reflexões nos profissionais, sobretudo, na equipe de enfermagem, que é a que, diante de suas atividades, permanece maior tempo próximo ao binômio familiar-doente. Por isso, tem demandado novas formas de organização, na dinâmica do cuidado em enfermagem, em grande parte pelos novos desafios enfrentados pelas famílias ${ }^{(14)}$.

Entretanto este cuidado que engloba a criança e sua família precisa ser ampliado de forma diferenciada, com foco nas atitudes das equipes, envolvendo a participação das famílias nos cuidados às crianças e não sendo vistas pela equipe de enfermagem apenas como coadjuvantes ${ }^{(15,16)}$.

As doenças crônicas resultam em maior tempo de hospitalização e atendimentos hospitalares mais frequentes, aumentando o estresse e vulnerabilidade nas famílias.Por outro lado, aproximam a família e a equipe de saúde, favorecendo a identificação das demandas de apoio ao atendimento às crianças, assim como a boa comunicação entre acompanhantes e equipe de enfermagem, a qual, mediante uma competência ética, otimiza o processo de enfrentamento da doença ${ }^{(14,17-19)}$.

A falta de informação dos profissionais desencadeia nas mães sentimentos de incertezas e dúvidas, que pode diminuir, desde que a equipe de enfermagem escute as queixas, temores das 
famílias, incluindo-as nos cuidados, melhorando, assim, a relação de confiança entre a equipe e a família $^{(9,20)}$

Ademais, a crescente ênfase à família, no contexto hospitalar, traz preocupações em como ela é percebida pela equipe de saúde, pensandoa como um sistema que não pode ser dissociado no cuidado à criança hospitalizada com câncer. 0 paciente infantil precisa da família de forma ativa e integrada nos cuidados, ajudando-o a enfrentar e adaptar-se a esse momento.

Tendo em vista a incidência progressiva do câncer infantil, a atuação da enfermagem vem se tornando cada vez mais significativa, já que seu trabalho é baseado na identificação das respostas humanas, visto que busca estabelecer estratégias que possam recuperar a saúde e o bem-estar da criança doente e de sua família.

À luz dessas considerações, este estudo tem como objetivo analisar a produção científica sobre a família da criança hospitalizada com câncer.

\section{MÉTODOS}

Trata-se de revisão integrativa da literatura (RIL), uma análise de pesquisas relevantes que permite a síntese dos resultados, viabilizando conclusões gerais, a respeito de uma área particular de estudo, assim como mostra lacunas do conhecimento que precisam ser preenchidas com novas pesquisas ${ }^{(21)}$.

Realizou-se, criteriosamente, o percurso metodológico, seguindo as seguintes etapas: formulação e identificação do problema e pergunta de pesquisa; estabelecimento dos critérios de inclusão e exclusão; coleta de dados; avaliação dos estudos incluídos; análise e

Quadro 1- Estratégia de busca

Quadro 1- Estratégia de busca
\begin{tabular}{|c|l|}
\hline Base de dados & \multicolumn{1}{c|}{ Estratégia de busca } \\
\hline BVSLILICS/BDENF & (tw:(Child* OR Crianca OR Nino OR Adolescent* OR Teen*)) AND (tw:(Neoplas* OR Tumor* OR Tumour* OR \\
& Cancer OR Carcinoma* OR Onco*)) AND (tw:(Family OR Familia OR Parent*)) AND (tw:("Nursing care" OR \\
& "Cuidados de Enfermagem" OR "Cuidado de Enfermeria" OR Nurs* OR Enfermeria OR Enfermagem)) AND \\
& (instance:"regional") AND (db:("LILACS") AND year_cluster:("2013" OR "2014" OR "2015" OR "2016" OR "2017" \\
& OR "2018")) \\
\hline PUBMED & $(((($ Child[mj] OR Child*[tiab] OR Adolescent[mj] OR Adolescent*[tiab] OR Teen*[tiab]) AND (Neoplasms[mj] OR \\
& Neoplas*[tiab] OR Tumor*[tiab] OR Tumour*[tiab] OR Cancer[tiab] OR Carcinoma*[tiab] OR Onco*[tiab])) AND \\
& (Family[mj] OR Family[tiab] OR Parents[tiab])) AND (Nursing care[mj] OR Nurs*[tiab])) AND ("2013/11/29"[PDat] \\
& $:$ "2018/11/27"[PDat] AND "humans"[MeSH Terms]) \\
\hline CINAHL & ((((Children OR Adolescents OR Teenager) AND (Neoplasms OR Tumor* OR Tumour* OR Cancer OR Carcinoma \\
& OR Oncology)) AND (Family OR Parents)) AND (“Nursing care" OR Nursing)) \\
\hline
\end{tabular}

interpretação dos dados; e apresentação dos resultados $^{(22)}$.

A elaboração da questão norteadora foi baseada na estratégia PICO, que fornece informações sobre o foco, escopo e aplicabilidade em uma revisão. Detalhando a estratégia PICO, o $P$ determina a população a ser estudada; o I a intervenção, a indicação ou interesse do que se deseja estudar; o C é a comparação e, por fim, O são o desfecho e o resultado esperado ${ }^{(23)}$.No acrônimo PICO desta revisão,serão investigados a perguntaPI, cujo $P$ é o familiar da criança hospitalizada com câncer e o I que identificados indicativos científicos publicados na literatura sobre a vivência familiar da criança hospitalizada com câncer.Emerge, assim, a seguinte questão norteadora: quais as evidências científicas sobre a vivência familiar da criança hospitalizada com câncer?

A busca foi realizada,durante os meses de janeiro a fevereiro de 2019, nas seguintes bases de dados: Literatura Latino-americana e do Caribe em Ciências da Saúde (LILACS), Medical Literature Analysis and Retrieval System On-line (MEDLINE), Cumulative Index to Nursing and Allied Health (CINAHL) eBase de Dados em Enfermagem (BDENF), cujo acesso foi feito por um único pesquisador. Com a finalidade de ampliar o campo de busca, foram diversificadas as bases de dados, atendendo à literatura nacional e internacional.

Os descritores utilizados na pesquisa foram identificados, nos Descritores em Ciências da Saúde (DECS), específicos das bases de dados LILACS, no Medical Subject Headings (MeSH) e por títulos, na base de dados CINAHL, respeitando as especificidades de cada um. O cruzamento dos descritores foi de acordo com os operadores boleanos AND e OR especificados no quadro 1 
Para a seleção dos artigos, foram definidos, como critérios de inclusão, artigos originais, nos idiomas Inglês, Português e Espanhol que retratavam a vivência familiar da criança com câncer no hospital, publicados entre2010 e 2019. Foram excluídos os artigos repetidos, nas bases de dados, estudos de revisão, relatos de casos, reflexões ou comunicação e artigos nos quais não foi possível identificar relação com a temática, por meio da leitura de título e resumo.

Nesta revisão integrativa, optou-se pela categorização de evidências fundamentada por especialistas da Agency for Healthcare Research and Quality (AHRQ), a saber: nível 1, revisão sistemática ou metanálise de ensaios clínicos randomizados controlados; nível 2, evidência de um ensaio clínico randomizado controlado e bem delineado; nível 3, ensaio clínico bem descrito, sem randomização; nível 4, estudo de coorte e caso-controle bem delineados; nível 5, revisões sistemáticas de estudos descritivos e qualitativos; nível 6, estudos descritivos e qualitativos e nível 7, opinião de autoridades respeitáveis, baseada na competência clínica ou opinião de comitês de especialistas ${ }^{(25)}$.

Sobre o grau de recomendação das evidências científicas, essa classificação denomina os níveis I e II como fortes, III e IV moderados e V a VII fracos, visto que a evidência nível I é a mais confiável e a melhor para responder à sua questão clínica ${ }^{(25)}$.Foram respeitadas todas as questões éticas e, por ser tratar de uma revisão integrativa, não houve necessidade de submissão ao Comitê de Ética em Pesquisa (CEP).

$\mathrm{Na}$ etapa de identificação, foram encontrados 413 artigos. Após leitura dos títulos e resumos, foram retirados 93 , na etapa de triagem, por não atenderem aos critérios de inclusão da pesquisa. Para a etapa de elegibilidade, foram recuperados 45 artigos préselecionados dentre os quais, na etapa de inclusão, dezenove compuseram a revisão, sendo retirados 23 artigos por não terem relação com o tema de estudo. 0 diagrama de fluxo, segundo o modelo meta-análise (PRISMA), encontra-se a seguir (Figura 2) (22)

Figura 2 - Fluxograma utilizado no processo de seleção dos artigos baseado no modelo PRISMA ${ }^{(22)}$.

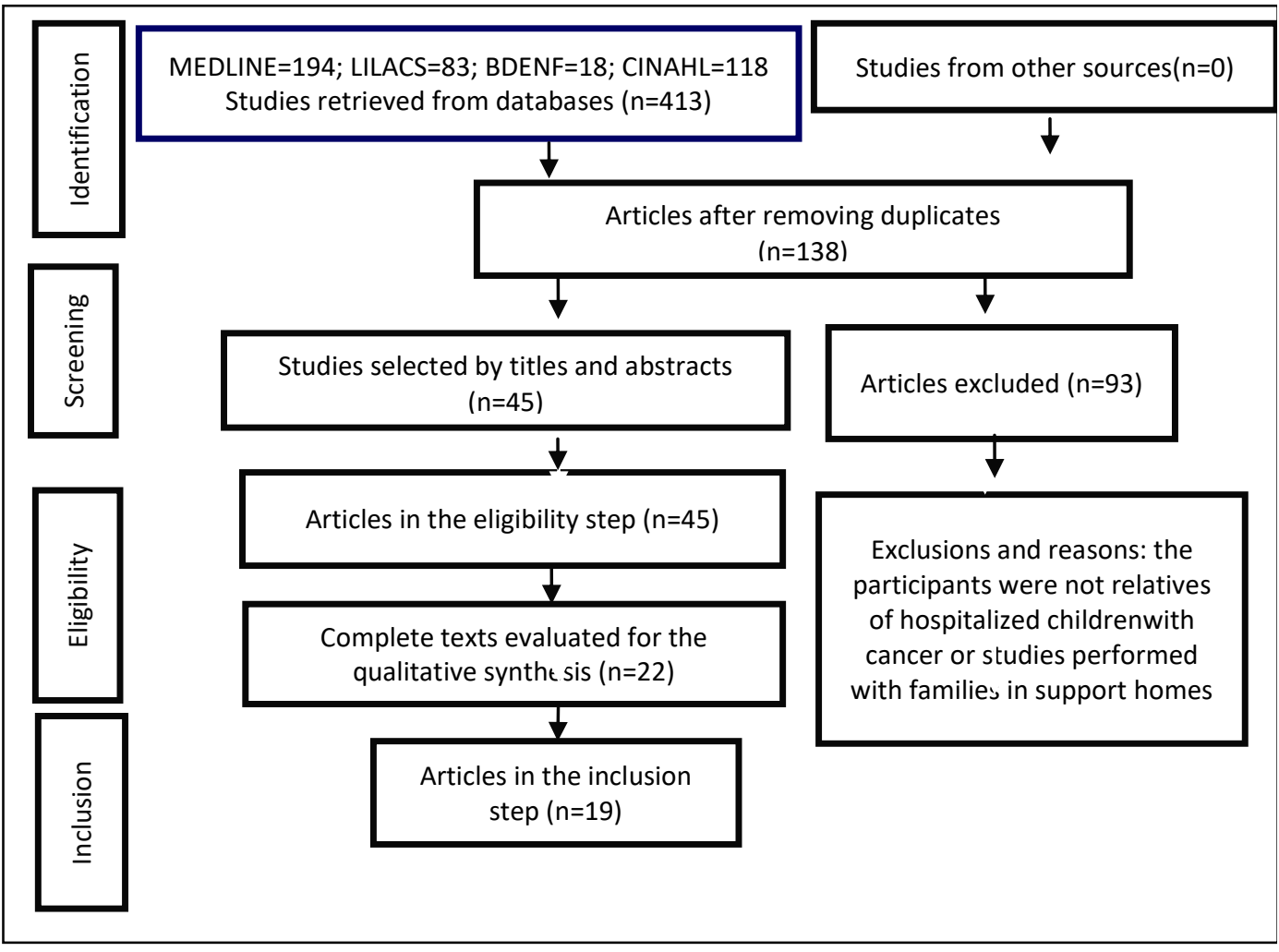


Os artigos da amostra final foram identificados com nome fictício por letras alfabéticas, seguindo a ordem da lista de referências apresentada nesta RIL submetidos a uma leitura exploratória, analisados na íntegra, de forma crítica e sistematizada.

Para garantir o rigor metodológico e verificar as evidências das publicações incluídas, as Figuras 2 e 3 foram elaboradas pelo autor, no Microsoft Word, especificando as principais informações dos artigos utilizados, identificados por letras alfabéticas, na ordem da lista de referências apresentada nesta $\mathrm{Rl}$,contemplando título, autores, ano, local de estudo, base de dados, nível de evidência e objetivos, facilitando a comparação dos estudos em tópicos específicos, para a análise e a redação do texto da revisão integrativa, discutidos à luz da literatura específica.

Por fim, os conteúdos das figuras permitiram, também, a organização dos dados e posterior sistematização dos resultados da busca, para melhor caracterização de todo o conteúdo coletado, seguindo as recomendações do modelo PRISMA ${ }^{(22)}$. Os estudos foram analisados por dois pesquisadores, de forma independente e interpretados com a perspectiva de atingir o objetivo desta pesquisa. A análise permitiu identificar as informações e as descrições mais frequentes e relevantes ao tema, que se destacaram quanto à intencionalidade da questão norteadora do presente estudo. Agruparam-se as seguintes categorias temáticas: "Sentimentos do familiar da criança hospitalizada com câncer. Necessidades do familiar da criança hospitalizada com câncer e Relevância da atuação da equipe de enfermagem".

Após a síntese dos dados extraídos dos artigos, a análise foi realizada de forma descritiva, com o intuito de reunir o conhecimento produzido sobre o tema explorado na revisão, respeitando todos os preceitos éticos e não havendo qualquer conflito de interesse.

\section{RESULTADOS E DISCUSSÃO}

De acordo com o ano de publicação, os dezenove artigos selecionados para o estudo, três $(15,79 \%)$ foram publicados nos anos de 2014 , 2016 e 2017; dois (10,53\%) em 2010, 2012, 2015 e 2018; 1(um) (5,26\%) em 2011 e 2013. Sobre os locais de estudos encontrados, o Brasil aparece com maior índice de publicação, quatorze $(73,68 \%)$; um $(5,26 \%)$ nos EUA; um $(5,68 \%)$ no Irã; um (5,26\%) na China; um $(5,26 \%)$ no Quênia e um $(5,26 \%)$ em Taiwan.

Em relação à base de dados, o CINAHL se destacou com $31,58 \%$ das publicações. No tocante ao delineamento da pesquisa, $100 \%$ utilizaram a abordagem qualitativa sendo 17 artigos categorizados, no nível de evidência 6, definido como estudos com delineamento não experimental, como pesquisa descritiva correlacional e qualitativa; e dois estudos foram encontrados, no nível de evidência 3, classificado como estudo com delineamento quase experimental, sem randomização com grupo único pré e pós-teste, séries temporais ou casocontrole de acordo com a classificação da Agency for Healthcare Research and Quality (AHRQ) ${ }^{(22)}$. Portanto, há evidência científica desta temática no nível de evidência6.

Figura3 -Caracterização dos artigos selecionados, conforme título, autor, ano de publicação, local de estudo, base de dados e nível de evidência científica.

\begin{tabular}{|c|c|c|c|c|c|c|}
\hline ID & TíTULO & AUTOR & ANO & LOCAL & $\begin{array}{l}\text { BASE DE } \\
\text { DADOS }\end{array}$ & $\begin{array}{l}\text { NÍVEL DE } \\
\text { EVIDÊNCIA }\end{array}$ \\
\hline A & $\begin{array}{l}\text { Mães/acompanhantes de crianças com } \\
\text { câncer: apreensão da cultura hospitalar }\end{array}$ & Vieira RFC et AL & 2017 & $\begin{array}{c}\text { Rio de Janeiro, } \\
\text { Brasil }\end{array}$ & LILACS & 6 \\
\hline B & $\begin{array}{l}\text { A vivência familiar diante do } \\
\text { adoecimento e tratamento de crianças } \\
\text { e adolescentes com leucemia linfoide } \\
\text { aguda }\end{array}$ & $\begin{array}{l}\text { Monteiro CFS, } \\
\text { Veloso LUP, } \\
\text { Sousa PCB, } \\
\text { Morais SCRV }\end{array}$ & 2014 & Teresina, Brasil & MEDLINE & 6 \\
\hline $\mathrm{C}$ & $\begin{array}{l}\text { Pediatric Oncology: the use of cluster } \\
\text { anallysis to examine maternal concerns }\end{array}$ & Liu YM, Yeh CH & 2010 & $\begin{array}{l}\text { Norte de } \\
\text { Taiwan } \\
\end{array}$ & CINAHL & 3 \\
\hline $\mathrm{D}$ & $\begin{array}{l}\text { Tensão do papel de cuidador principal } \\
\text { diante do cuidado prestado a crianças } \\
\text { com câncer }\end{array}$ & $\begin{array}{c}\text { Oliveira RAA, et } \\
\text { AL }\end{array}$ & 2015 & $\begin{array}{c}\text { Pernambuco } \\
\text { Brasil }\end{array}$ & LILACS & 6 \\
\hline $\mathrm{E}$ & Dimensões da vulnerabilidade para as & Motta MGC, & 2013 & Rio Grande do & LILACS & 6 \\
\hline
\end{tabular}




\begin{tabular}{|c|c|c|c|c|c|c|}
\hline & $\begin{array}{l}\text { famílias da criança com dor oncológica } \\
\text { em ambiente hospitalar }\end{array}$ & DiefenbachGDF & & Sul, Brasil & & \\
\hline $\mathrm{F}$ & $\begin{array}{l}\text { Incertezas diante do câncer infantil: } \\
\text { compreendendo as necessidades da } \\
\text { mãe }\end{array}$ & Angelo $\mathrm{M}$, et $\mathrm{AL}$ & 2010 & São Paulo, Brasil & MEDLINE & 6 \\
\hline G & $\begin{array}{l}\text { O cotidiano dos pais de crianças com } \\
\text { câncer e hospitalizadas }\end{array}$ & $\begin{array}{l}\text { Duarte MLC, et } \\
\qquad \mathrm{AL}\end{array}$ & 2012 & $\begin{array}{l}\text { Rio Grande do } \\
\text { Sul, Brasil }\end{array}$ & BDENF & 6 \\
\hline $\mathrm{H}$ & $\begin{array}{l}\text { Apoio social entre acompanhantes de } \\
\text { crianças hospitalizadas em uma } \\
\text { unidade de hematopediatria }\end{array}$ & Kozan,L, et AL & 2016 & Curitiba, Brasil & LILACS & 6 \\
\hline I & $\begin{array}{l}\text { Ser mãe de criança com câncer: uma } \\
\text { investigação fenomenológica }\end{array}$ & Santos LF, et AL & 2011 & Goiás, Brasil & BDENF & 6 \\
\hline J & $\begin{array}{l}\text { Segredos e verdades no processo } \\
\text { comunicacional da família com a } \\
\text { criança com câncer }\end{array}$ & $\begin{array}{l}\text { Borges AA, } \\
\text { DuppasG }\end{array}$ & 2016 & $\begin{array}{l}\text { Minas Gerais, } \\
\text { Brasil }\end{array}$ & LILACS & 6 \\
\hline $\mathrm{L}$ & $\begin{array}{l}\text { How Parents of Children With Cancer } \\
\text { Learn About Their Children's Prognosis }\end{array}$ & Sisk BA et AL & 2018 & $\begin{array}{c}\text { Boston, } \\
\text { Massachusetts } \\
\text { Pensilvânia }\end{array}$ & MEDLINE & 3 \\
\hline$M$ & $\begin{array}{l}\text { Psychological Limbo as a Barrier to } \\
\text { Spiritual Care for Parents of Children } \\
\text { with Cancer: A Qualitative Study }\end{array}$ & $\begin{array}{l}\text { Sheikhzakaryae } \\
\text { e, Neda, et AL }\end{array}$ & 2018 & Irã & MEDLINE & 6 \\
\hline $\mathrm{N}$ & $\begin{array}{l}\text { Coping with the diagnosis and } \\
\text { hospitalization of a child with } \\
\text { childhood cancer }\end{array}$ & NoiaTC, et AL & 2015 & Bahia, Brasil & MEDLINE & 6 \\
\hline 0 & $\begin{array}{l}\text { Female caregivers accompanying } \\
\text { children with cancer in the hospital } \\
\text { setting }\end{array}$ & $\begin{array}{l}\text { Wegner W, } \\
\text { Pedro ENR. }\end{array}$ & 2017 & $\begin{array}{l}\text { Rio Grande do } \\
\text { Sul, Brasil }\end{array}$ & BDENF & 6 \\
\hline$P$ & $\begin{array}{l}\text { Psychometric Validation of an } \\
\text { Instrument to Measure Family Coping } \\
\text { During a Child's Hospitalization for } \\
\text { Cancer }\end{array}$ & Liu $Q$, et $A L$ & 2017 & China & CINAHL & 6 \\
\hline$Q$ & $\begin{array}{l}\text { Repercussions of child cancer in the } \\
\text { daily life of a family caregiver } \\
\text { Repercussões }\end{array}$ & Medeiros, et AL & 2014 & Paraíba, Brasil & CINAHL & 6 \\
\hline $\mathrm{R}$ & $\begin{array}{l}\text { Assessment of Parents' Perception of } \\
\text { Quality of Pediatric Oncology Inpatient } \\
\text { Care at Kenyatta National Hospital }\end{array}$ & $\begin{array}{l}\text { Keiza EM, } \\
\text { Chege MN, } \\
\text { Omuga BO }\end{array}$ & 2016 & Quênia & CINAHL & 6 \\
\hline$S$ & $\begin{array}{l}\text { O cuidar em enfermagem: família e } \\
\text { criança com dor oncológica }\end{array}$ & $\begin{array}{l}\text { Diefenbach } \\
\text { GDF, Motta } \\
\text { MGC }\end{array}$ & 2012 & $\begin{array}{l}\text { Rio Grande do } \\
\text { Sul, Brasil }\end{array}$ & CINAHL & 6 \\
\hline $\bar{T}$ & $\begin{array}{l}\text { Reactions of family members of } \\
\text { children diagnosed with cancer: a } \\
\text { descriptive study }\end{array}$ & $\begin{array}{c}\text { Carvalho AS, } \\
\text { Depianti JRB, } \\
\text { Silva LF, Aguiar } \\
\text { RCB, Monteiro } \\
\text { ACM }\end{array}$ & 2014 & $\begin{array}{c}\text { Rio de Janeiro, } \\
\text { Brasil }\end{array}$ & CINAHL & 6 \\
\hline
\end{tabular}

Fonte: Elaborado pelos autores.

Os estudos revelaram a assertiva de que a equipe de enfermagem é peça fundamental de elo entre a díade família e criança hospitalizada, devendo promover educação em saúde, ao mesmo tempo em que realiza a assistência, combinando compreensão, habilidades técnicas, humanização e sensibilidade no cuidado.

A primeira categoria temática foi construída, com base em dezoito artigos, ressaltando o sentimento de medo, dor, tristeza, ansiedade e vulnerabilidade, enfatizado pelo impacto do diagnóstico, do desconhecido, das incertezas e da possibilidade da perda e morte $^{(26,29-37)}$.
As famílias esquecem suas próprias vidas, anulam-se, quando têm um filho em tratamento de câncer. Não projetam seus futuros pelo medo da perda; seu cotidiano famílias passa a ser o das crianças. Elas saem das suas próprias histórias e mergulham nas histórias de vida de seus filhos, servindo-Ihes de sustento ${ }^{(26,33,39)}$.

Ocorrem, na vida de mães de crianças com câncer, diferentes alterações no campo pessoal e profissional, tais como desemprego, enfraquecimento do relacionamento conjugal, isolamento social, desgaste físico e emocional, em virtude de uma necessidade de reorganização para cuidar do filho doente ${ }^{(40)}$. 
Esses sentimentos de medo e insegurança nos familiares, durante a hospitalização da criança com câncer, são ocasionados pela fragilidade psicológica aliada à gravidade da doença e às incertezas do tratamento, que desenvolvem angústias, desestabilizam as famílias e refletem na qualidade de vida, sendo diretamente proporcionais, ocasionando tensão ao cuidador ${ }^{(30,42)}$.

O câncer associado à pessoa jovem traz reflexões quanto à possibilidade de futuro, podendo frustrar projetos de vida, principalmente, diante da ameaça de morte. Então, algumas estratégias surgem quanto ao diagnóstico, como a de não querer falar sobre o câncer, ao assumir uma postura de negação para minimizar o sofrimento ${ }^{(43)}$.

A vulnerabilidade está associada ao impacto do diagnóstico; o estar doente e a hospitalização, para a criança e sua família com internações freqüentes alteram o seu cotidiano e passam a vivenciar um novo contexto, imerso em incompreensões com relação ao cuidado, ao sofrimento gerado pelo tratamento e pela dor oncológica ${ }^{(30-31,39,44)}$.

O impacto do diagnóstico de câncer reflete nas famílias a idéia de uma doença ainda muito estigmatizada e sem cura, causando perda rápida e morte inevitável, porém a forma de lidar com as mudanças, a hospitalização e a doença estão intimamente relacionadas às características próprias de cada família ${ }^{(28,31,33,45)}$.

Por outro lado, foi possível também identificar que as mães se tornam fortes, diante das interações, adaptando-se ao ambiente, encorajando-se durante os procedimentos hospitalares, conseguindo dominar seus medos, fortalecendo inclusive outras mães que vivenciam o mesmo contexto. Culturalmente essas mães são vistas como fortes, guerreiras e têm oportunidades de crescimento com a doença, que propicia oportunidade de compreensão mútua ${ }^{(26,39,42)}$.

A possibilidade da perda, diante do diagnóstico do câncer, causa nas famílias uma experiência de sentimentos intensos que perpassam pelas fases da doença, sempre com o risco iminente de uma perda precoce tornandoas famílias vulneráveis. Para as mães, o diagnóstico de câncer contribui para impactos negativos, como sentença de morte ${ }^{(33-34,45)}$.

Conviver com o desconhecido resulta em sentimentos de incertezas e inseguranças que fazem parte do universo do tratamento oncológico, cujo sucesso de toda a trajetória está diretamente ligado à forma como essa fase será vivida por todos ${ }^{(32,39)}$.

A segunda categoria trata-sedas necessidades do familiar da criança com câncer. Emergiu, em dez artigos, o desejo de estar presente, acompanhar o tratamento, receber uma comunicação clara, ser ouvida, amparada, resgatar a espiritualidade para a melhora da qualidade de vida e manter vínculos ${ }^{(26,30,33,35-39,45}$ 46).

O fato de estar presente e acompanhar o tratamento do filho proporcionam às mães segurança, para estabelecer vínculos de confiança com a equipe, que trata e cuida do seu filho. Essa mãe necessita de apoio e acolhimento para não desenvolver o sentimento de solidão(33).

É a mãe ou outro parente do sexo feminino quem mais acompanha a criança na hospitalização, sendo responsável por manter vínculo de confiança com a equipe de saúde, organizando formas de enfrentamento, almejando a cura e o retorno à normalidade da dinâmica familiar ${ }^{(32,37)}$.

A forma de comunicação clara demonstrou ser forte aliada ao tratamento da criança hospitalizada e essencial ao cuidado, pois é extremamente eficiente na assistência de enfermagem aos familiares da criança com câncer. Quando informados dos prognósticos, tornam-se mais confiantes e otimistas ${ }^{(27,36,45)}$.

Mediante as demandas da doença e hospitalização, tanto criança quanto familiar necessitam ser ouvidos e amparados pela equipe, que deve otimizar espaços de acolhimento para que a mães possam expressar suas emoções, a fim de lhes proporcionar sua inserção no cuidado de seu filho ${ }^{(30,39)}$.

Ao atender as necessidades do familiar, amparando e, sobretudo, ouvindo seus anseios e sentimentos, os profissionais de saúde têm nas famílias uma forte parceira no cuidado ao paciente, com capacidade de reformular as informações dadas no hospital e agregá-las ao cotidiano do cuidado ${ }^{(26,35)}$.

A terceira categoria aborda enfermagem e família no enfrentamento da doença. Foi evidenciada, em dezessete artigos, a relevância da atuação da equipe de enfermagem junto as famílias na detecção de possíveis problemas, identificando o risco de enfrentamento ineficaz dessas famílias diante da doença e suporte 
emocional, buscando amenizar o cotidiano por meio de uma comunicação clara e resgate da autonomia ${ }^{(26-33,36-46)}$.

Outro aspecto mostrado nesta categoria foi referente às interações entre enfermagem e família, no ambiente hospitalar, que reduzem os fatores estressores no que diz respeito ao diagnóstico, hospitalização e tratamento da doença. Sendo assim, trazem conforto e estreitam vínculos, auxiliando no enfrentamento da doença e em suas dificuldades, neste período crítico, ao oferecer uma assistência humanizada ${ }^{(34,44)}$.

A equipe de enfermagem são os primeiros a estabelecer contato com o momento inicial da família, no ambiente hospitalar. Além disso, é a categoria que mais tempo permanece junto à família e à criança, durante a hospitalização, criando mais vínculos. Dessa forma, deve estar apta a perceber dificuldades e intervir com segurança adequada, assegurando o suporte emocional às famílias durante este momento ${ }^{(28,31)}$.

Ressalta-se que quanto mais se incentivar a participação dos familiares da criança, tornandoos importantes e indispensáveis no cuidado, inserindo-os nas rotinas diárias, nas soluções de problemas e conflitos, mais fácil será transformar a relação intersubjetiva mais sólida, além de ajudar no resgate da autonomia, da qualidade de vida e enfrentamento da doença ${ }^{(33)}$.

Constatou-se que a ausência de informação aumenta a dificuldade de saber mais sobre a doença, agravando os sentimentos de impotência e tristeza. O profissional de enfermagem deve entender a família, como uma unidade de cuidado, que faz parte do tratamento da criança, pois a doença está relacionada com a interrupção do percurso da vida ${ }^{(34,46)}$.

Quanto mais se reforçam as informações pela enfermagem, maior o apoio, o acolhimento, estabelecimento de vínculo e confiança, dando suporte ao familiar, pois será significativo ao processo de cuidar. $O$ diálogo transmite segurança e continuidade do tratamento em domicílio $^{(47)}$.

Uma forma de amenizar sintomas de ansiedade, preocupação e medo, diante do tratamento do câncer nas famílias, está na busca da espiritualidade que traz tranquilidade e a capacidade de resistir aos problemas catastróficos, pois a fé em Deus reduz o pensamento negativo e tem um importante papel no equilíbrio emocional, sendo uma estratégia adotada pelas acompanhantes das crianças com câncer ${ }^{(33,37,39)}$.

A confiança em Deus, a fé e a espiritualidade são ferramentas que ajudam no processo de aceitação da doença, fortalecem, encorajam, melhoram a expectativa de cura e possibilitam meios para reestruturarem suas famílias ${ }^{(48-49)}$.

No cuidado à criança oncológica, os profissionais devem considerar as relações interpessoais, com zelo e competência técnica, durante todo o processo, a fim de diminuir as consequências do tratamento e da hospitalização para a criança e sua família ${ }^{(38,50-51)}$.

Diante das possibilidades desta revisão, evidenciou-se que as pesquisas sobre as famílias foram desenvolvidas, buscando a investigar as experiências dos familiares, considerando as situações de sofrimento, desgaste físico e emocional, alterações nas rotinas, necessidades das famílias de serem apoiadas, ouvidas, compartilharem pensamentos, formarem vínculos afetivos, estarem presentes, durante o tratamento, diante do medo, da insegurança, da angústia e do novo contexto vivido no diagnóstico do câncer.

Todavia, a partir da análise das publicações, foi possível constatar que a enfermagem possui relevante papel no cuidado às famílias, durante a hospitalização, visto que representam apoio e suporte à criança com câncer, que, no momento de fragilidade, torna-se vulnerável e perde sua autonomia. Deste modo, o cuidado não pode ser desvinculado da díade criança e família.

Neste sentido, torna-se necessária a reflexão sobre a importância de a enfermagem inserir o cuidado centrado na família, no tratamento da criança oncológica, apoiando-se em teorias de enfermagem, englobando as diversas interfaces que a hospitalização traz para a criança e seu familiar, respeitando e valorizando os sentimentos, necessidades e as limitações de forma individual e humana.

\section{CONCLUSÃO}

Foram encontradas evidências científicas sobre a família da criança com câncer,destacando-se as dificuldades em receber o diagnóstico de câncer em um filho, revelando uma experiência de dor, desespero, medos, questionamentos, inseguranças, ansiedade e 
muita preocupação com o futuro incerto. Destaca-se o papel relevante da enfermagem, na hospitalização da criança e em sua situação, pois se trata de um ambiente desconhecido, cercado de aparelhagem e novas rotinas. Neste cenário, a enfermagem deve contribuir com ações que atendam às dimensões clínicas, afetivas, sociais e emocionais das famílias, que precisam ser amparadas e acolhidas, para resgatarem sua autonomia; construir essas relações de confiança, fortalecendo-as para o enfrentamento da doença.

Os achados nesta revisão reforçam ainda mais o papel fundamental da enfermagem no cuidado centrado na criança e em sua família diante do diagnóstico de câncer. A família deve ser vista como uma unidade de cuidado que precisa ser ouvida, acolhida e ensinada para dar continuidade ao tratamento da criança. A interação entre família e equipe de enfermagem constrói vínculos, relações de confiança e segurança no cuidado.

Uma limitação deste artigo foi a escassez de publicações com a temática família da criança hospitalizada, especificamente com câncer, mesmo tendo sido constatados aumentos expressivos de artigos publicados, com ênfase na família, a partir da promulgação do Estatuto da Criança e do Adolescente, em 1990.

Verificou-se, também, a necessidade de mais estudos, que apresentem melhor classificação quanto ao grau dos níveis de evidências em relação à temática família da criança com câncer, que apontem resultados mais precisos do cuidado centrado a família. Do mesmo modo, poderão auxiliar futuras pesquisas que incorporem as famílias, nas atuações do cuidado, trazendo melhores indicadores e fácil acesso a resultados que fundamentam condutas e tomadas de decisões de enfermagem na assistência à criança com câncer e à sua família no cenário hospitalar.

\section{REFERÊNCIAS}

1- Paixão TM, Farias SNP, Rosas AMMTF, Coropes BVAS. Early detection and child cancer approach for primary care.Rev Enferm UFPE2018;12(5):1437-43. DOI:10.5205/19818963-v12i5a231281p1437-1443-2018 Disponível em:https://periodicos.ufpe.br/revistas/revistaenf ermagem/article/view/231281.

2- Fidler MM, Bray F. Global cancer inequalities.Front Oncol. 2018;8:293. DOI:

\subsection{9/fonc. 2018.00293}

3- Instituto Nacional de Câncer José Alencar Gomes da Silva. Estimativa 2020: Incidência de câncer no Brasil. Rio de Janeiro: INCA; 2019.

4- Costa AR, Nobre CMG, Gomes GC, Nornberg PKO, Rosa GSM. Sentimentos gerados na família pela internação hospitalar da criança. J Nurs Health 2019;9(2):1-12. DOI: 10.15210/jonah.v9i2.14012

5- Turolla KR, Souza MC. Enfermagem Pediátrica Oncológica: Assistência na fase de terminalidade. EnsaiosCiênc,CiencBiol Agrar Saúde 2015 [citado em 7abr 2019];19(1):26-37.Available in: file://C:/Users/FAMILIA/Downloads/3013-

Texto\%20do\%20artigo-12380-1-10-20151119.pdf 6- Leite AO, Medeiros CCM, Medeiros DDM, Batista DA. Ansiedade do acompanhante diante da assistência de enfermagem na ala de pediatria. Temas Saúde 2017 [citado 25 abr 2019]; 17(1):147-66. Available in: http://temasemsaude.com/wpcontent/uploads/2017/05/17110.pdf

7- Paula DPS, Silva GRC, Andrade JMO, Paraiso AF. Câncer infanto-juvenil do âmbito familiar: Percepções e experiências frente ao diagnóstico. RevCuid. 2019; 10(1):1-12. DOI: 1015649/cuidarte.v10il.571

8- Gomes GC, Xavier DM, Pintanel AC, Farias DHR, Lunardi VL, Aquino DR. Significados atribuídos por familiares na pediatria acerca de suas interações com os profissionais da enfermagem. RevEscEnferm USP2015;49(6):9539. DOI:10.1590/S0080-623420150000600011

9- Chagas MCS, Gomes GC, Pereira FW, Diel PKV, Farias DHR. Significado atribuído a família ao cuidado da criança hospitalizada. AvEnferm. 2017;35(1):7-18.DOI:

10.15446/av.enferm.v35n1.42466

10- Morais RCM, Souza TV, Oliveira ICS, Moraes RMM. A estrutura da rede social da mãe/acompanhante da criança hospitalizada. CogitareEnferm. 2018;1(23):1-10.DOI: 10.5380/ce.v23i1.50456

11- Fernandes MA, Soares AJ, Ferraz MMM, Silva FAA, Silva JS, Carvalho RJ. Representações sociais por mães com filhos em tratamento de câncer. RevEnferm Atual. 2019 [citado 25 abr 2019]; 89(27):1-7. Available in: https://revistaenfermagematual.com.br/index.ph $\mathrm{p} /$ revista/article/view/418/487

12- Neutzling BRS, Barlem JGT, Barlem ELD, Hirsch CD, Pereira LA, Schallenberguer CD. Em defesa dos direitos da criança no ambiente 
hospitalar: O exercício da advocacia em saúde pelos enfermeiros. Esc Anna Nery Rev Enferm. 2017;21(1):1-9. DOI: $10.5935 / 1414-$ 8145.20170025

13- Passos SSS, Henckemaier L, Costa JC, Pereira A, Nitschke RG. Daily care of families in hospital: What about patient safety? TextoContextoEnferm. 2016;25(4):1-10.DOI: 10.1590/010407072016002980015

14- Silva TP, Leite JL, Stinson J, Laloo C, Silva IR, Jibb L. Estratégias de ação e interação para o cuidado à criança hospitalizada com dor oncológica crônica. Texto Contexto-Enferm. 2015;27(4):1-11. DOI: 10.1590/010407072018003990017

15- Neves L, Gondim AA, Soares SCMR, Coelho $D P$, Pinheiro JAM. O impacto do processo de hospitalização para o acompanhante familiar do paciente crítico crônico internado em uma unidade de terapia semi-intensiva. Esc Anna Nery 2018;22(2):1-8.DOI: $\quad$ 10.1590/2177-9465-EAN2017-0304

16- Azevêdo AVS, Lançoni Júnior AC, Crepaldi MA. Interação equipe de enfermagem, família, e criança hospitalizada: Revisão integrativa. Ciênc Saúde Coletiva 2017;22(112):3653-66. DOI: 10.1590/1413-812320172211.26362015

17- Machado NA, Nóbrega VM, Silva MEA, França DBL, Reichert APS, Collet N. Doença crônica infanto-juvenil: Vínculo profissionalfamília para a promoção do apoio social. RevGaúcha Enferm. 2018;39:1-8. DOI: 10.1590/1983-1447.2018.2017-0290

18- Ferreira LB, Oliveira JSA, Gonçalves RG, Elias TMN, Medeiros SM, Mororó DDS. Cuidar de Enfermagem às famílias de crianças e adolescentes hospitalizados. Rev Enferm UFPE 2019;13(1):23-31. DOI: 10.5205/1981-8963v13i01a237672p23-31-2019

19- Fontes ALC, Patricio ACFA, Lima TDS, Nascimento LBM, Silva RAR. Vulnerabilidade ao estresse: Pais cuidadores de filhos com câncer. Cuidado e Fundamental. RevFun Care 2019;11(4):857-64.DOI:10.9789/2175-

5361.2019.v11i4.857-861

20- Santos RS, Takeshita IM, Araujo CM, Jardim ASL, Cunha GR. Percepção das mães de crianças com câncer sobre o cuidado humanizado da enfermagem. RevEnfermCent-Oeste Min. 2019; 9:e2883. DOI: 10.19175/recom.v9i0.2883

21- Ercole FF, Melo LS, Alcoforado CLGC. Integrative review versus systematic review. Rev Min Enferm. 2014;18(1):9-12. DOI:
10.5935/1415-2762.20140001

22- Galvão TF, Pansani TSA. Principais itens para relatar Revisões Sistemáticas e Meta-análises: A recomendação do PRISMA. EpidemiolServ Saúde 2015;24(2):1-8.

DOI:10.5123/S1679-

\section{7}

23- The Joanna Briggs Institute. Joanna Briggs Institute Reviewers' Manual: 2015 edition. 2015[citado 15 mar 2020]. Available in: https:// https://nursing.Isuhsc.edu/JBI/docs/ReviewersM anuals/Scoping-.pdf

24- Pompeo DA, Rossi LA, Galvão CM. Revisão integrativa: Etapa inicial do processo de validação de diagnóstico de enfermagem. Acta Paul Enferm. 2009;22(4): DOI: 434-8. 10.1590/S010321002009000400014

25- Melnyk BM, Fineout-Overholt E. Evidence based practice in nursing \& healthcare: A guide to best practice. 2nd ed. Philadelphia: Wolters Kluwer Health; 2011.

26- Vieira RF, Souza TV, Oliveira ICS, Morais RCM, Gois JR. Mothers/companions of children with cancer: Apprehension of the hospital culture.Esc Anna Nery 2017;21(1):1-7. DOI: 10.5935/1414-8145.20170019

27- Borges AA, Lima RAG, Dupas G. Segredos e verdades no processo comunicacional da família com a criança com câncer. Esc Anna Nery 2016;20(4):1-9.DOI:10.5935/1414-

8145.20160101

28- Monteiro CFS, Veloso LUP, Souza PCB, Coelho $S$, Morais RV. A vivência familiar diante do adoecimento e tratamento de crianças e adolescentes com leucemia linfoide aguda. CogitareEnferm $2008 \quad$ [citado 25 Fev2019];13(4):484-9. Available in: https://revistas.ufpr.br/cogitare/article/view/131 04/8863

29- Liu YM, Yeh CH. Pediatric Oncology: The use of cluster analysis to examine maternal concerns. OncolNurs Forum 37(4):4-11. DOI: 10.1188/10.ONF.E304-E311

30- Oliveira RAA, Moura TML, Perreli JGA, Lopes MVO, Oliveira SM. Tensão do papel do cuidador principal diante do cuidado prestado a crianças com câncer. RevCuba Enferm. 2015 [citado 20 mar 2020]; 31(2). Available in: www.revenfermeria.sld.cu/index.php/enf/article/ view/331/125

31- Motta MGC, Diefenbach GDF. Dimensions of vulnerability for the Family of the child with oncologic pain in the hospital environment. Esc 
Anna Nery 2013; 1(3):482-90. DOI:10.1590/S1414-81452013000300011

32- Angelo M, Moreira PL, Rodrigues LMA. Incertezas diante do câncer infantil: Compreendendo as necessidades da mãe.Esc Anna Nery 2019;14(2):301-8. DOI: 10.1590/S1414-81452010000200013

33- Duarte MLC,Zanini LN, Nede MNB.O cotidiano dos pais de criança com câncer e hospitalizadas. RevGaúchEnferm. 2012;33(3): 111-8. DOI: 10.1590/S1983-14472012000300015 34- Alves KMC, Comassetto I, Almeida TG, Treza MCSF, Silva JMO, Magalhães APN. The experience of parentes of children with cancer in treatment failure conditions. Texto Contexto-Enferm. 2016;25(2):1-9. DOI: 10.1590/010407072016002120014

35- Kozan L, Wanderbroocke ACNS, Polli GM. Apoio social entre acompanhantes de crianças hospitalizadas em uma unidade de hematopediatria. PsicolHosp. 2016 [citado 10 mar 2019]; 14(1):53-78. Available in: http://pepsic.bvsalud.org/scielo.php?script=sci_a bstract\&pid=S1677-

74092016000100004\&lng=pt\&nrm=iso

36- Santos LF, Marinho KC, Oliveira RR, Siqueira KM, Oliveira LMAC, Peixoto MKAV, et al. Ser mãe de criança com câncer: Uma investigação fenomenológica. RevEnferm UERJ2011 [citado 10 mar 2019];19(4):626-31. Available in: http://www.facenf.uerj.br/v19n4/v19n4a21.pdf 37- Sheikjzakaryaee N, Atashzadeh-Shoorideh F, Ahmadi F, Fani M. Psychological limbo as barrier to spiritual care for parentes of children with câncer: A qualitative study. Asian Pac J Cancer Prev. 2018;19(4):1063-68. DOI: 10.22034/APJCP.2018.19.4.1063

38- Noia TC, Sant'ana SER, Santos ADS, Oliveira SC, Veras SMCB, Lopes-Junior CL. Coping with the diagnosis and hospitalization of a child with childhood cancer. Invest EducEnferm. 2015;33(3):465-72.

DOI:

10.17533/udea.iee.v33n3a10

39- Wegner W, Pedro ENR. Female caregivers accompanying children with cancer in the hospital setting. RevGaúcha Enferm. 2010;31(4):678-84. DOI: 10.1590/S198314472010000400010

40- Costa MADJ, Agra G, Souza Neto VL, Silva BCO, Laísi CBB, Mendonça AEO. Desvelando a experiência de mães de criança com câncer. 2016; 1(6):2052-65.DOI:
41- Lyu QY, Kong SKF, Wong FKY, You LM, Yan J, Zhou $X Z$, et al. Psycometric validation of na instrument to measure Family coping during a child's hospitalization for cancer. Cancer Nurs. 2017;40(3):194-200.

DOI:10.1097/NCC.0000000000000382

42- Medeiros EGMS, Leite RFB, Ramos DKR, Almeida LAL. Repercussions of child cancer in the daily life of a family caregiver. Rev Rene2014;15(2):233-9. DOI:10.15253/21756783.2014000200007

43- Karkow MC, Perlini NMOG, Stamm B, Camponogara S, Terra MG, Viero V. Experience of families facing the revelation of the câncer diagnosis in one of its integrants. Rev Min Enferm. 2015;19(3):747-51. DOI:0.5935/14152762.20150056

44- Diefenbach GD, Motta MGC. O cuidar em enfermagem: Família e criança com dor oncológica.CogitareEnferm. 2012;17(3):458-63. DOI:10.5380/ce.v17i3.24752

45- Carvalho AS, Depianti JRB, Silva LF, Aguiar RCB, Monteiro ACM. Reações da família da criança com diagnóstico de câncer: Estudo descritivo. Braz J Nurs. 2014 [citado 31 maio 2019]; 13(3):282-91. Available in: https://pesquisa.bvsalud.org/portal/resource/pt/ biblio875771http://www.objnursing.uff.br/index. php/nursing/article/view/4356/pdf_173

46- Sisk BA, Kang TI, Mack JW. How parents of children with cancer learn about their children's prognosis.

Pediatrics2018;141(1):1-10. DOI:10.1542/peds.2017-2241

47- Rodrigues JRG, Siqueira Júnior AC, Siqueira FPC. Nursing consultation in pediatric oncology: $A$ tool for empowering parentes. RevFun Care 2020;12:202-11. DOI: 10.9789/21755361.rpcfo.v12.7569

48- Vidotto PCP, Ferrari RAP, Tacla MTGM, Facio $B C$. Experiência materna no itinerário diagnóstico do câncer infantil. RevEnferm UFPE 2017;11(4):1565-73. DOI:10.5205/reuol.976385423-1-SM.1104201702

49- Alves DA, Silva LG, Delmondes GA, Lemos ICS, Kerntopf MR, Albuquerque GA. Cuidador da criança com câncer: Religiosidade e espiritualidade como mecanismos de enfrentamento. RevCuid. 2016;7(2):1318-24. DOI: 10.15649/cuidarte.v7i2.336

50- Keiza EM, Chege MN, Omuga BO. Assessment of parents' perception of quality of pediatric oncology inpatient care at 
keniattanational hospital. Asia Pac J OncolNurs. 2017;4(1):29-37. DOI:10.4103/2347-5625.199071

51- Hopia H, Tolonen TH. Families in paediatric oncology nursing: Critical incidents from the nurses perspective. J PediatrNurs.2019;44:28-35. DOI: 10.1016/j.pedn.2018.10.013

Nota: Este estudo está articulado ao Projeto de Doutorado do Programa de Pós Graduação em Ciências do Cuidado em Saúde, linha de pesquisa de Cuidados Clínicos da Escola de Enfermagem Aurora de Afonso Costa da Universidade Federal Fluminense.

Recebido em: 28/10/2019

Aprovado em: 30/03/2020

\section{Endereço de Correspondência:}

Rua Garibaldi, 225 bloco II, 1109, Tijuca, Rio de Janeiro, Brasil. CEP: 20511-330 